\title{
Elastic limit angular speed of solid and annular disks under thermo- mechanical loading
}

\author{
Priyambada Nayak ${ }^{1}$, Kashinath Saha ${ }^{2 *}$ \\ ${ }^{1,2}$ Department of Mechanical Engineering, Jadavpur University, Kolkata-700032, INDIA \\ "Corresponding Author: e-mail: kashinathsaha@gmail.com,Tel +91-332-4572659, Fax. +91-332-4137121
}

\begin{abstract}
In this study, the influence of thermo-mechanical loading on stresses and deformation states in a rotating disk with varying thicknesses has been evaluated based on a variational principle considering the radial displacement field as unknown. The solutions till the elastic limit yield stress of the material are attained with the assumption of plane stress condition. MATLAB ${ }^{\circledR}$ computational simulation software is used to implement the solution algorithm. The analysis is accomplished for different disk geometries as well as temperature distribution profiles. Limit angular speed of the disks is calculated under thermo-mechanical loading and accounted for in dimensional mode as limit peripheral speed and dimensionless form as normalized limit angular speed. The influence of temperature on yield stress and subsequently on the limit peripheral speed is also studied for any given temperature distribution and results are furnished in dimensional form. The effects of temperature field on other material properties are also studied. The results of some reduced problems are validated with those available in literature and very good agreement is observed. The new results, furnished graphically as design monograms, might prove helpful for the practicing engineers.
\end{abstract}

Keywords: Rotating disks, Variational method, Thermo-elastic stresses.

\section{Introduction}

Rotating disks are vital parts of diverse mechanical applications, for instance disk brakes, flywheels, gears, circular saws, hard disks, gas as well as steam turbine rotors, internal combustion engines, centrifugal compressors, and in aerospace industries. Mechanical design of disks entails the assessment of centrifugal as well as thermal stresses and they need to be designed for approximate uniform stress distributions. The analyses of stresses and strains of these rotating elements have been studied by several authors and investigators. Various types of theoretical, semi-analytical or numerical approaches are employed to solve complicated problems involving diverse thickness profiles, variation in mechanical as well as physical properties and boundary conditions, etc. The behavior of rotating disks under high temperature has investigated by Thompson (1946) in which he gave a numerical method to the turbine disk problem by accounting for point-to-point variations in disk thickness and in all other physical properties apart from Poisson's ratio.

In structural design process, it is expected that the estimation of the stress distribution and the angular velocity of a rotating disk in a fully-plastic state is important and found momentum in 1980s where several researchers employed the Tresca yield criterion. Gamer (1983) pointed out that the displacement field calculated according to Tresca's criterion as well as its related flow rule at the elasto-plastic interface of a rotating disk, was discontinuous and a negative plastic strain caused by a tensile stress. To work out this problem, the author Gamer $(1984,1985)$ advanced an extra linear strain hardening for a rotating disk of constant thickness of elastic plastic material having a state of plane stress. Rees (1999) applied von Mises criteria and its associated flow rule to avoid the insufficiency of Tresca solution in the elasto-plastic stress distribution of rotating disk by using a numerical iteration method. 
Tresca's yield condition has been found to predict slightly lower limit angular velocities than that of von Mises. The idea was extended by Eraslan (2003) in presenting an analytical solution for elastic-plastic deformation of rotating variable thickness annular disks with free, pressurized as well as radially-constrained boundary conditions.

Analysis of elastic stress state in rotating disks up to the point of yielding with the objective to determine limit angular speed has also been carried out recently. Vivio and Vullo (2007) as well as Vullo and Vivio (2008) have studied the stresses and strains in variable thickness annular and solid rotating elastic disks under the influence of thermal loads as well as containing a variable density all along the radius. Hojjati and Jafari (2008) reported approximate solutions of similar problems appearing as an infinite power series for nonlinear equations, by using Adomian's decomposition method (ADM). Bayat et al. (2008) obtained solutions for the elastic and thermo-elastic problems for functionally graded (FG) rotating disks under the assumption that the material properties as well as disk thickness varies following power-law functions of radius. The axisymmetric displacements as well as stresses in functionally graded hollow cylinders, disks and spheres under the influence of uniform internal pressure, using plane elasticity theory and 'Complementary Functions' method, has been proposed by Tutuncu and Temel (2009). Bhowmick et al. (2010) studied the limit angular speed of externally loaded rotating disks subject to shrink-fit by using variational method. In a subsequent paper, Bhowmick et al. (2010) also investigated the development of elastic-plastic front in rotating solid disks of nonuniform thickness with exponential as well as parabolic geometry variation by extending a variational method in elastoplastic regime.

Axisymmetric thermo-elastic analysis of a rotating radially-graded hollow circular disk under the influence of radial temperature distribution was conducted by Peng and Li (2010) using one-dimensional elasticity theory. Nie and Batra (2010) considered stresses in isotropic, linear thermo-elastic incompressible functionally graded rotating disks of variable thickness. Closed-form solutions are presented wherever possible; otherwise the governing equations are solved by differential quadrature method. Rattan et al. (2010) investigated steady-state creep response of an isotropic FGM rotating disc of aluminum silicon carbide particulate composites and considered creep in the presence of thermal residual stress as well. Wauer and Schweizer (2010) studied the dynamic thermo-elastic problems of rotating disks with stationary sources of heat and forces at small, bordered areas of the surface.

A thorough literature review in this research area reveals that analyses of problems are carried out mostly through analytical methods and no dedicated work on limit angular speed of rotating disks under thermo-mechanical loading has been carried out. The principal goal of this research is to study the effect of thermo-mechanical loading on stresses and deformation states in a rotating disk with varying thicknesses. For this purpose, a numerical method based on variational principle has been proposed to formulate the problem and to achieve an estimated solution of the unknown displacement field from the governing equation. MATLAB® computational simulation software is used to implement the solution algorithm. The results reported by various other researchers are validated and good agreement is obtained. The results furnished in the present study would provide significant understanding of the behavior of rotating disks under thermo-mechanical loading. Furthermore, the effect of disk geometry and temperature field variation on the performance of rotating disks is considered and normalized value of limit angular speed is furnished.

\section{Mathematical Formulation}

In the development of the mathematical model, it is assumed that the disk material is homogeneous, isotropic and linear elastic. For the problem at hand, a response analysis of thermo-elastic stresses is studied under the structure of small deformation. The disk is thin, symmetric with respect to the mid-plane and plane stress $\left(\sigma_{z}=0\right)$ assumption is justified.

Radial displacements will occur in a rotating disk, due to the both centrifugal load and thermal load. Besides the magnitude of loading, the radial displacement field is also governed by the boundary conditions of the disk. The result for the displacement field is found from the minimum total potential energy principle, $\delta(U+V)=0$, where $U$ is the strain energy stored in the disk and $V$ is the potential energy evolving both from centrifugal force as well as thermal load. It is already stated that the material of the disk is isotropic, so the thermal strain at any location of the disk is same in all the directions. If $\alpha$ is thermal expansion coefficient and $T(r)$ is the change in temperature at any radius $r$, then the thermal strain is given by $\varepsilon^{*}=\alpha T(r)$.

The total strain is obtained by adding the elastic strain and the thermal strain. Thus, the components of the total strain are given by $\varepsilon_{r}=e_{r}+\varepsilon^{*}$ and $\varepsilon_{\theta}=e_{\theta}+\varepsilon^{*}$, where $\varepsilon_{r}$ and $\varepsilon_{\theta}$ denote the radial and circumferential components, respectively, with respect to the total strain and $e_{r}$ and $e_{\theta}$ are the radial and circumferential components of the elastic strain. The elastic strain components are related to stresses by Hooke's law and thus the expression of total strain components become, $\varepsilon_{r}=\left(\sigma_{r}-v \sigma_{\theta}\right) / E+\alpha T(r)$ and $\varepsilon_{\theta}=\left(\sigma_{\theta}-v \sigma_{r}\right) / E+\alpha T(r)$, where $E$ and $v$ are elastic modulus and Poisson's ratio. For axisymmetric problems with small strains, the relations between strains and radial displacement are given by $\varepsilon_{r}=d u / d r$ and $\varepsilon_{\theta}=u / r$. The total strain energy that comes from the stress and strain field of the disk, and expressed as 


$$
\Pi=\frac{1}{2} \int_{V o l}(\sigma \varepsilon) d v=\frac{1}{2} \int_{V o l}\left(\sigma_{r} \varepsilon_{r}+\sigma_{\theta} \varepsilon_{\theta}\right) d v
$$

Substituting the relations between stress-strain and strain-displacement, equation (1) becomes,

$$
\Pi=\frac{\pi E}{\left(1-v^{2}\right)_{a}^{b}} \int_{a}\left\{\frac{u^{2}}{r}+2 v u \frac{d u}{d r}+r\left(\frac{d u}{d r}\right)^{2}-(1+v) \alpha T r\left(\frac{u}{r}+\frac{d u}{d r}\right)\right\} h d r
$$

At this point, the total energy $\Pi$ is decomposed into two parts: A strain energy $U$ and the potential energy $V$. The first part of potential energy $V_{\text {centrifugal }}$ comes from centrifugal force field, while the second part $V_{\text {thermal }}$, comes from the thermal load. The expressions for $U, V_{\text {centrifugal }}$ and $V_{\text {thermal }}$ are given below.

$$
\begin{aligned}
& \left.U=\frac{\pi E}{\left(1-v^{2}\right)_{a}^{b}} \int_{a} \frac{u^{2}}{r}+2 v u \frac{d u}{d r}+r\left(\frac{d u}{d r}\right)^{2}\right\} h d r \\
& V_{\text {centrifugal }}=-2 \pi \rho \omega^{2} \int_{a}^{b} u r^{2} h d r \\
& V_{\text {thermal }}=-\frac{2 \pi E}{\left(1-v^{2}\right)}(1+v) \alpha \int_{a}^{b} r\left(\frac{u}{r}+\frac{d u}{d r}\right) T h d r
\end{aligned}
$$

So, the expression for potential energy $V$ under combined loading becomes,

$$
V=-2 \pi \rho \omega^{2} \int_{a}^{b} u r^{2} h d r-\frac{2 \pi E}{\left(1-v^{2}\right)}(1+v) \alpha \int_{a}^{b} r\left(\frac{u}{r}+\frac{d u}{d r}\right) T h d r
$$

On substitution of equations (3) and (6) in the energy principle $\delta(U+V)=0$, the governing equilibrium equation is obtained as,

$$
\delta\left[\frac{\pi E}{\left(1-v^{2}\right.} \int_{a}^{b}\left\{\frac{u^{2}}{r}+2 v u \frac{d u}{d r}+r\left(\frac{d u}{d r}\right)^{2}\right\} h d r-2 \pi \rho \omega^{2} \int_{a}^{b} u r^{2} h d r-\frac{2 \pi E(1+v) \alpha^{b}}{\left(1-v^{2}\right)} \int_{a}^{b} r\left(\frac{u}{r}+\frac{d u}{d r}\right) T h d r\right]=0
$$

Equation (7) is stated in normalized co-ordinate, $\xi(=(r-a) /(b-a))$ and using the notation $\bar{r}=b-a$, it takes the following form,

$$
\begin{aligned}
& \delta\left[\frac{E \bar{r}}{\left(1-v^{2} \int_{0}^{1}\right.} \int_{0} \frac{u^{2}}{(\bar{r} \xi+a)}+\frac{2 v u}{\bar{r}}\left(\frac{d u}{d \xi}\right)+\frac{(\bar{r} \xi+a)}{\bar{r}^{2}}\left(\frac{d u}{d \xi}\right)^{2}\right\} h d \xi-2 \rho \omega^{2} \bar{r} \int_{0}^{l}(\bar{r} \xi+a)^{2} u h d \xi \\
& \left.-\frac{2 E(1+v) \alpha \bar{r}^{l}}{\left(1-v^{2}\right)} \int_{0}^{1}(\bar{r} \xi+a)\left(\frac{u}{(\bar{r} \xi+a)}+\frac{d u}{\bar{r} d \xi}\right) T h d \xi\right]=0
\end{aligned}
$$

The boundary conditions of the displacement function $u(\xi)$ for a solid disk are $\left.u\right|_{(0)}=0$ and $\left.\sigma_{r}\right|_{(b)}=0$, whereas for an annular disk, these conditions are $\left.\sigma_{r}\right|_{(a)}=0$ and $\left.\sigma_{r}\right|_{(b)}=0$. The necessary displacement function employed to produce the higher order orthogonal functions that satisfies above boundary conditions are given below, for solid and annular disks.

$$
\phi_{o}(r)=\frac{\rho \omega^{2} r(3+v)}{8 E}\left[b^{2}(1-v)-\left\{\frac{\left(1-v^{2}\right) r^{2}}{(3+v)}\right\}\right]+\frac{\alpha}{r}\left[(1+v) \int_{a}^{r} T(r) r d r+\frac{\left(1-v^{2}\right) r^{2}}{b^{2}} \int_{a}^{b} T(r) r d r\right]
$$




$$
\begin{aligned}
& \phi_{o}(r)=\frac{\rho \omega^{2} r(3+v)}{8 E}\left[\left(b^{2}+a^{2}\right)(1-v)-\left\{\frac{\left(1-v^{2}\right) r^{2}}{(3+v)}\right\}+b^{2} a^{2} \frac{(1+v)}{r^{2}}\right] \\
& +\frac{\alpha}{r}\left[(1+v) \int_{a}^{r} T(r) r d r+\frac{\left(1-v^{2}\right) r^{2}+(1+v) a^{2}}{b^{2}-a^{2}} \int_{a}^{b} T(r) r d r\right]
\end{aligned}
$$

For computational purpose, the set of orthogonal functions are employed to estimate the displacement function $u(\xi)$ as follows,

$$
u(\xi) \cong \sum c_{i} \phi_{i}, i=1,2, \ldots, n
$$

Function $\phi_{o}(r)$ is normalized and the governing equation in matrix form is obtained by substituting equation (10) in equation (8).

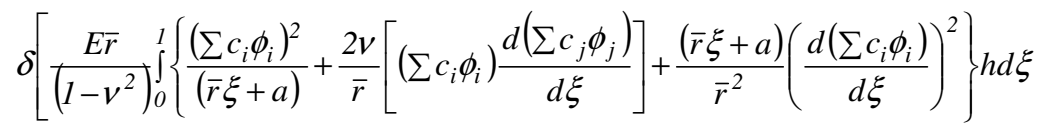

$$
\begin{aligned}
& \left.-2 \rho \omega^{2} \bar{r} \int_{0}^{l}\left\{(\bar{r} \xi+a)^{2}\left(\sum c_{j} \phi_{j}\right)\right\} h d \xi-\frac{2 E \alpha \bar{r}}{(1-v)} \int_{0}^{1}\left\{\left(\sum c_{j} \phi_{j}\right)+\frac{(\bar{r} \xi+a)}{\bar{r}} \frac{d\left(\sum c_{j} \phi_{j}\right)}{d \xi}\right\} T h d \xi\right]=0
\end{aligned}
$$

Replacing operator ' $\delta$ ' in equation (11) by $\partial / \partial c_{j}, j=1,2, \ldots, n$, the governing yields in agreement with Galerkin's error minimization principle as,

$$
\begin{aligned}
& \frac{E \bar{r}}{\left(1-v^{2}\right)} \sum_{i=1}^{n} \sum_{j=1}^{n} c_{i} \int\left\{\frac{\phi_{i} \phi_{j}}{(\bar{r} \xi+a)}+\frac{v}{\bar{r}}\left(\phi_{i}^{\prime} \phi_{j}+\phi_{i} \phi_{j}^{\prime}\right)+\frac{(\bar{r} \xi+a)}{\bar{r}^{2}} \phi_{i}^{\prime} \phi_{j}^{\prime}\right\} h d \xi \\
& =\rho \omega^{2} \bar{r} \sum_{j=1}^{n} \int_{0}^{l}\left\{(\bar{r} \xi+a)^{2} \phi_{j}\right\} d \xi+\frac{E \alpha}{(1-v)} \sum_{j=1}^{n} \int_{0}^{l}\left\{\phi_{j} \bar{r}+(\bar{r} \xi+a) \phi_{j}^{\prime}\right\} h d \xi
\end{aligned}
$$

where ( ) indicates differentiation with respect to normalized coordinate $\xi$. Equation (12) can be expressed as $[K]\{c\}=\{f\}$, which yields the solution vector $\left\{c_{i}\right\}$ through a single step matrix inversion process. The problem considered in the present paper is von-Mises yield criterion under plane stress can be written in the form

$$
\sigma_{v m}^{2}=\sigma_{r}^{2}-\sigma_{r} \sigma_{\theta}+\sigma_{\theta}^{2} \geq \sigma_{y}^{2}
$$

From the solution vector of equation (12), the resulting displacement field is post-processed to determine the von-Mises stress corresponding to the thermal and centrifugal loading. The loading is initiated at low values and increments are provided until the condition of yielding is reached.
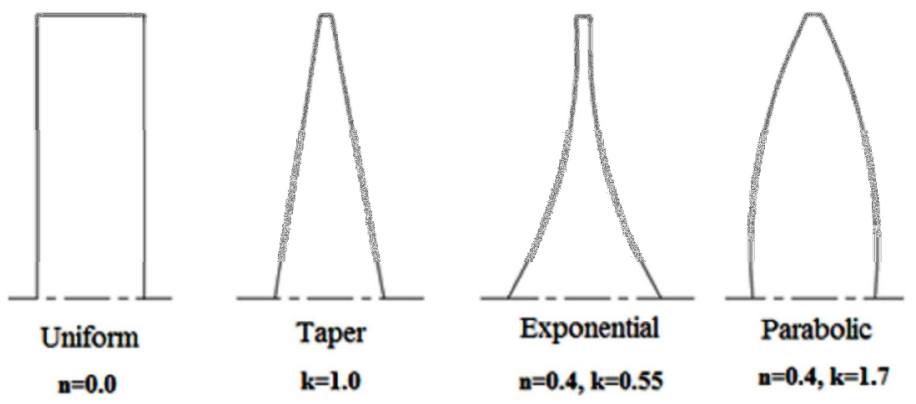

Figure 1. Thickness profiles of disk corresponding to geometric parameters $n, k$.

\section{Results and Discussions}

The effect of temperature on the limit angular speed for any given temperature distribution and boundary conditions are established in the present study. The analysis is carried out for four different profiles of disk, uniform, taper, exponential and 
parabolically varying thickness. The expression for an exponentially varying disk given by $h(\xi)=h_{0} \exp \left[-n(\xi)^{k}\right]$, whereas for a parabolically varying disk the expression becomes, $h(\xi)=h_{0}\left[1-n(\xi)^{k}\right]$, where $h_{0}$ indicates disk thickness at the inner radius of the disk. With the form of disk profile function for parabolic thickness variation, a uniform thickness disk is obtained by setting $n=0$ and a linearly varying thickness (taper) is obtained by setting $k=1$.

The profiles considered in this study are derived by using constant volume criteria, which would help to characterize the performance of the disk. The thickness of the uniform disk is taken as 5\% of its outer radius and corresponding to this volume, other disk profiles are calculated. It is further assumed that for disks of varying thickness, the tip thickness is $1 \%$ of outer radius. This particular assumption is indeed necessary to determine the geometry parameters $n$ and $k$, as shown in Figure 1.
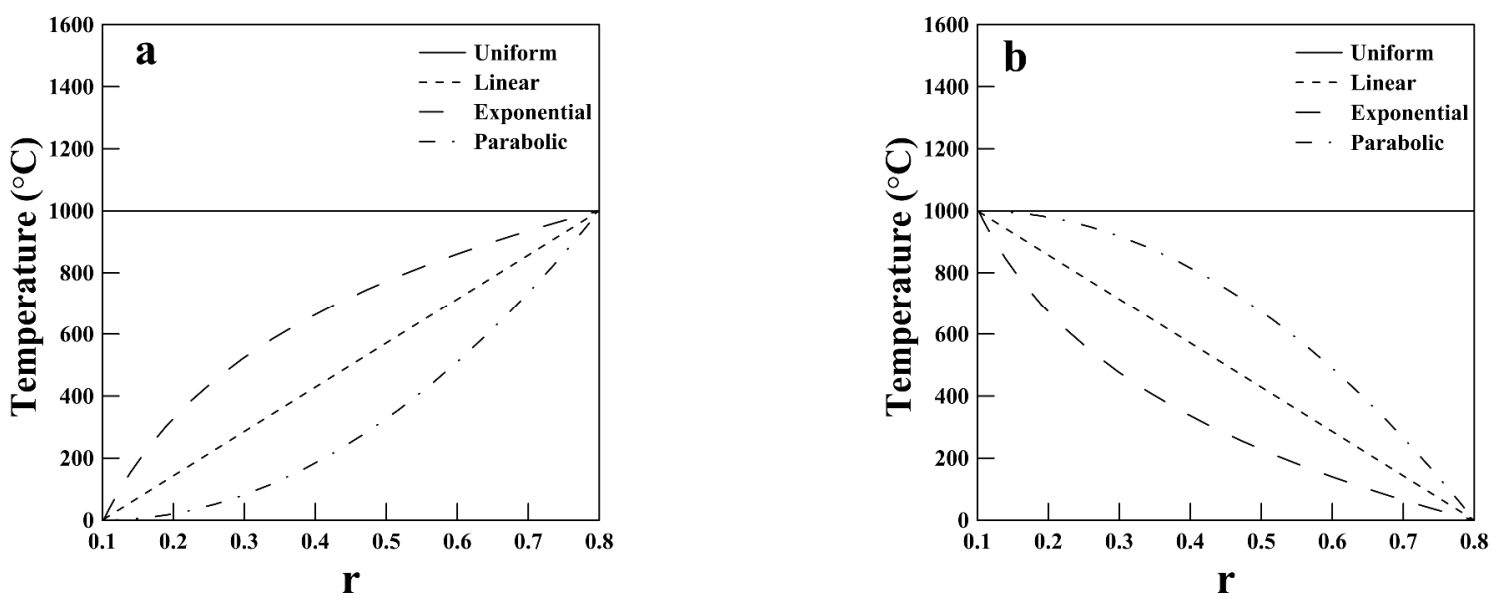

Figure 2. Rotating disk under various temperature distribution profiles for (a) specified outer surface temperature $\left(T_{b}\right)$, and (b) specified inner surface temperature $\left(T_{a}\right)$.

The dimensionless angular speed, $\omega_{y} b \sqrt{\rho / \sigma_{y}}$ corresponding to the onset of yielding is defined as normalized limit angular speed $\left(\bar{\omega}_{y}\right)$ and considered as the design parameter for disks under uniform temperature environment. The analysis is also carried out for various varying temperature profiles following uniform, linear, exponential and parabolic temperature distribution, as expressed in equation (14) and shown in Figure 2(a) and (b). It may be noted that the mathematical relations for the four types of temperature distributions are not identical with that of the thickness distribution relations.

$$
\begin{array}{llrl}
\text { Uniform: } & & T(r)=T_{a} \text { or } T_{b} \\
\text { Linear: } & & T(r)=T_{a}+\left(T_{b}-T_{a}\right) \xi \\
\text { Exponential: } & T(r)=\frac{\left(T_{b}-T_{a}\right) \ln (b / r)}{\ln (b / a)} \\
& \text { Parabolic: } & T(r)=T_{a}+\left(T_{b}-T_{a}\right) \xi^{2}
\end{array}
$$

The temperature field pattern is a function of temperature boundary conditions also. Two different, increasing and decreasing, temperature boundary conditions are assumed i) $T_{a}=0{ }^{\circ} \mathrm{C}, T_{b}=1000{ }^{\circ} \mathrm{C}$ and ii) $T_{a}=1000{ }^{\circ} \mathrm{C}, T_{b}=0{ }^{\circ} \mathrm{C}$, where $T_{a}$ and $T_{b}$ are the inner and outer surface temperature of the isotropic disk at $r=a$ and $r=b$ respectively. The temperature distribution profiles, shown in Figure 2(a) and (b), correspond to these two boundary conditions.

In the definition of dimensionless angular speed $\omega_{y} b \sqrt{\rho / \sigma_{y}}$, it is assumed that the disk material properties $E, \rho, \sigma_{y}, \alpha$ and $v$ remain constant in the variable thermal field $T(r)$. The preliminary part of the study is carried out based on this assumption but in the later part, when the disk material properties are assumed to be function of thermal field $T(r)$, dimensional value of limit angular tip speed, $\omega_{y} b$ in $(\mathrm{m} / \mathrm{s})$ is used as the design parameter.

The numerical analysis of the present study is carried out by considering system parameter values as, $E=210 G P a, v=0.3$, $\rho=7800 \mathrm{~kg} / \mathrm{m}^{3}, \quad \alpha=0.21 \times 10^{-6}{ }^{\circ} \mathrm{C}^{-1}$ and initial yield stress $\sigma_{y}=350 \mathrm{MPa}$. The number of coordinate functions used in the 
present study to approximate the displacement field, as expressed in equation (10), is 11. This particular number has been arrived at after carrying out the necessary convergence tests on normalized limit angular speed for a uniform disk. The validation plots for the displacement and stress (radial and tangential) field are presented in Figure 3(a) to (e) which shows that the agreement of the results of computation with the same using the results of Vivio and Vullo (2007) is very good. Detail discussions on these figures are reported in Vivio and Vullo (2007) and hence they are not repeated here to maintain brevity.

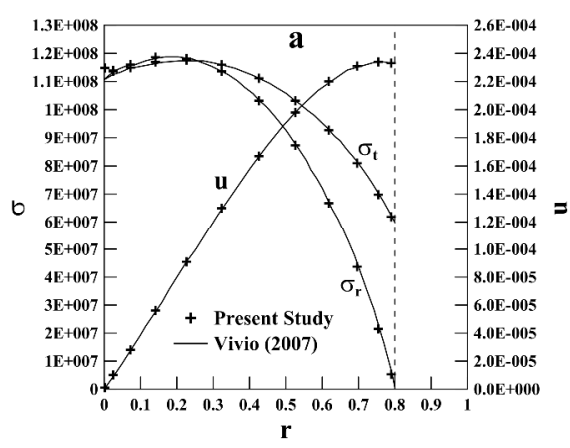

$\mathbf{r}$

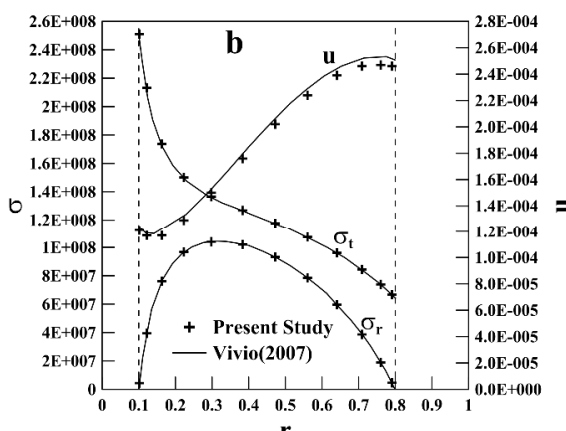

$\mathbf{r}$
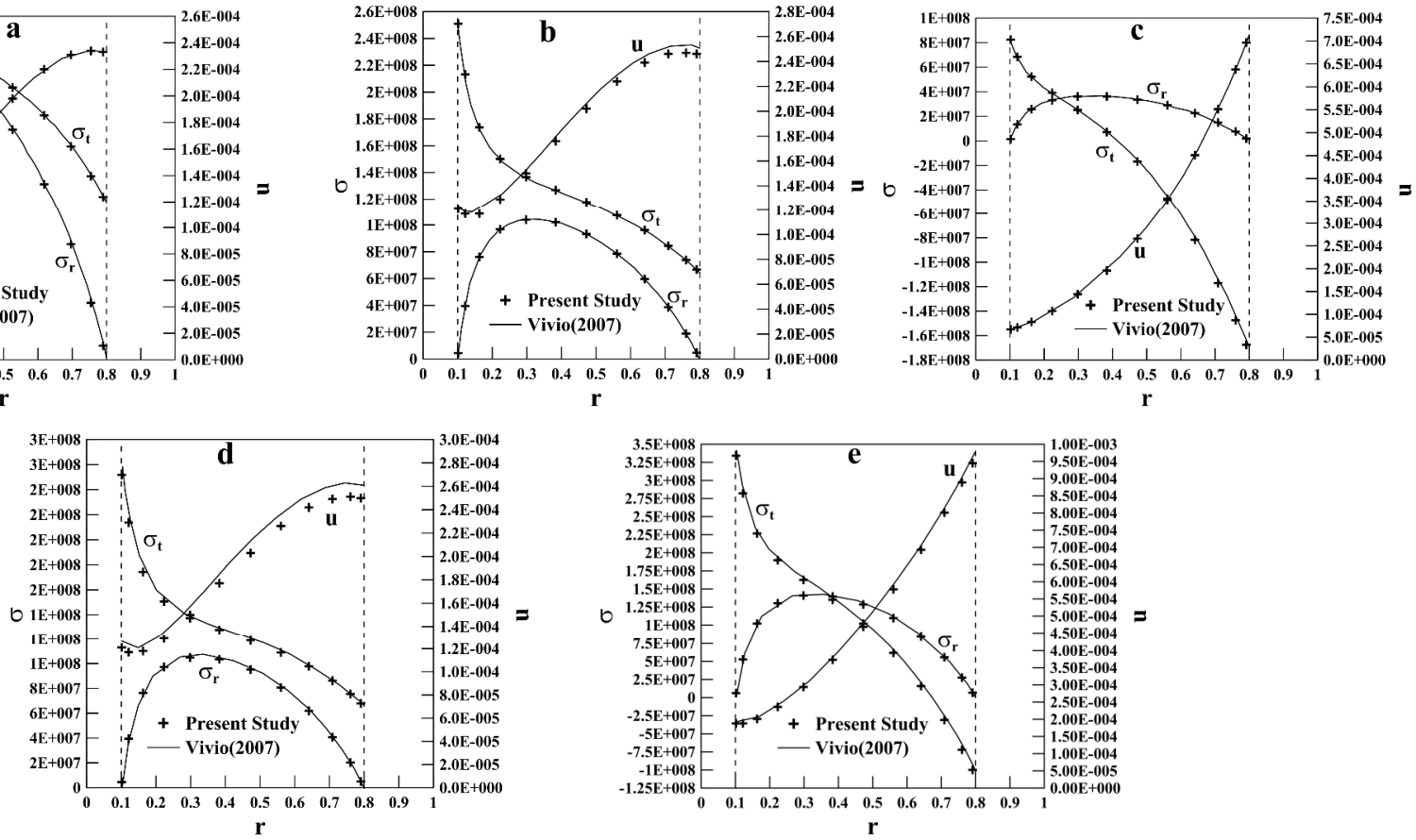

Figure 3. Comparison plots for displacement $(u)$, radial $\sigma_{r}$ and tangential stresses $\sigma_{t}$ with the results of Vivio and Vullo (2007) for: (a) solid taper disk, (b) annular taper disk, (c) annular taper disk subjected to temperature gradient and (d) annular taper disk with density variation (e) annular taper disk subjected to both density and temperature gradient.
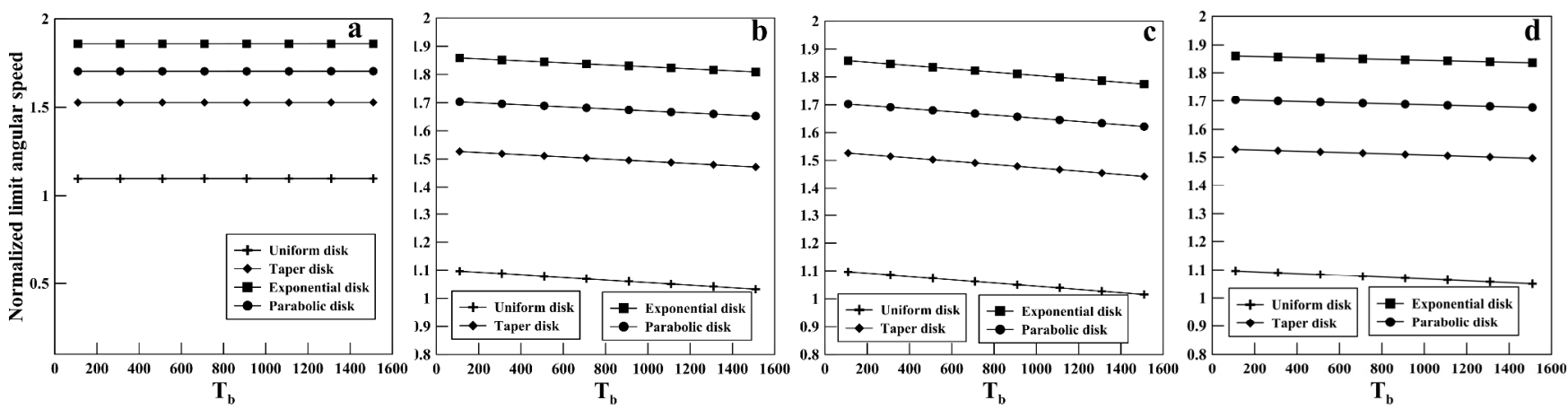

Figure 4. Plots for normalized limit angular speed $\left(\bar{\omega}_{y}\right)$ with outer surface temperature $\left(T_{b}\right)$ having different temperature distributions: (a) uniform, (b) linear, (c) exponential and (d) parabolic.

3.1 Effect of temperature distributions: The effect of temperature distributions $\left(T_{b}\right)$ on the normalized limit angular speed $\left(\bar{\omega}_{y}\right)$ is presented in Figure 4(a) to (d) for annular disks and in each figure, variation is shown for four different disk geometries. The inner surface temperature $T_{a}$ is set to $0^{\circ} \mathrm{C}$ and the aspect ratio of disk $(a / b)$ is taken as 0.8 . It is assumed that material properties $E, \alpha, \rho$ and $v$ remain constant in the variable thermal field of the disk. It is observed that with increase in outer surface temperature $T_{b}$, the normalized limit angular speed decreases for all type of disk geometries. However, for uniform temperature distribution the normalized limit angular speed remains constant. Amongst the different geometries, normalized limit angular speed is maximum for exponential disk geometry. Again, in case of exponential temperature distribution, the decrease of normalized limit angular 
speed is prominent for different disk geometries. It is observed in the analytical study that among all the physical parameters, coefficient of thermal expansion $\alpha$ has the predominant effect on the variation in $\bar{\omega}_{y}$.

A similar study on the normalized limit angular speed variation with temperature is indicated in Figure 5(a) to (d), for the prescribed disk geometries and temperature distributions. Here, the inner surface temperature $T_{a}$ is varied but the outer surface temperature $T_{b}$ is kept fixed at $0^{\circ} \mathrm{C}$. In Figure 5(a), the normalized limit angular speed is constant for uniform temperature distribution as that of the previous case while in Figure 5(b) to (d), the normalized limit angular speed increases with increase in inner surface temperature $T_{a}$ for different disk geometries for all types of temperature distributions. These figures show that the normalized limit angular speed is maximum for exponential disk geometry. The results also indicate that for exponential temperature distribution; the increase of normalized limit angular speed is prominent for different disk geometries.
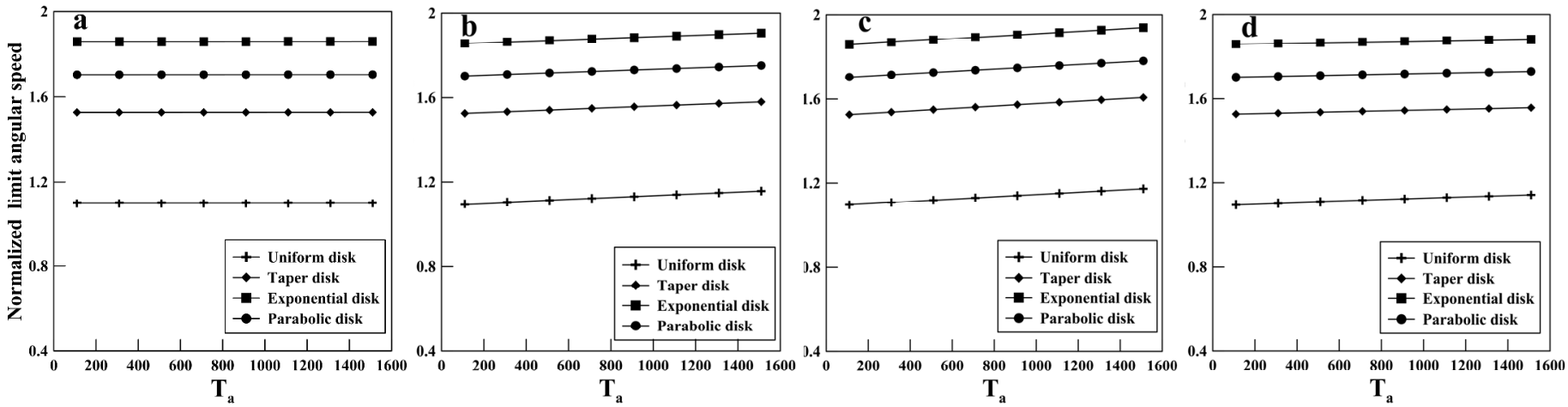

Figure 5. Plots for normalized limit angular speed $\left(\bar{\omega}_{y}\right)$ with outer surface temperature $\left(T_{a}\right)$ having different temperature distributions: (a) uniform, (b) linear, (c) exponential and (d) parabolic.
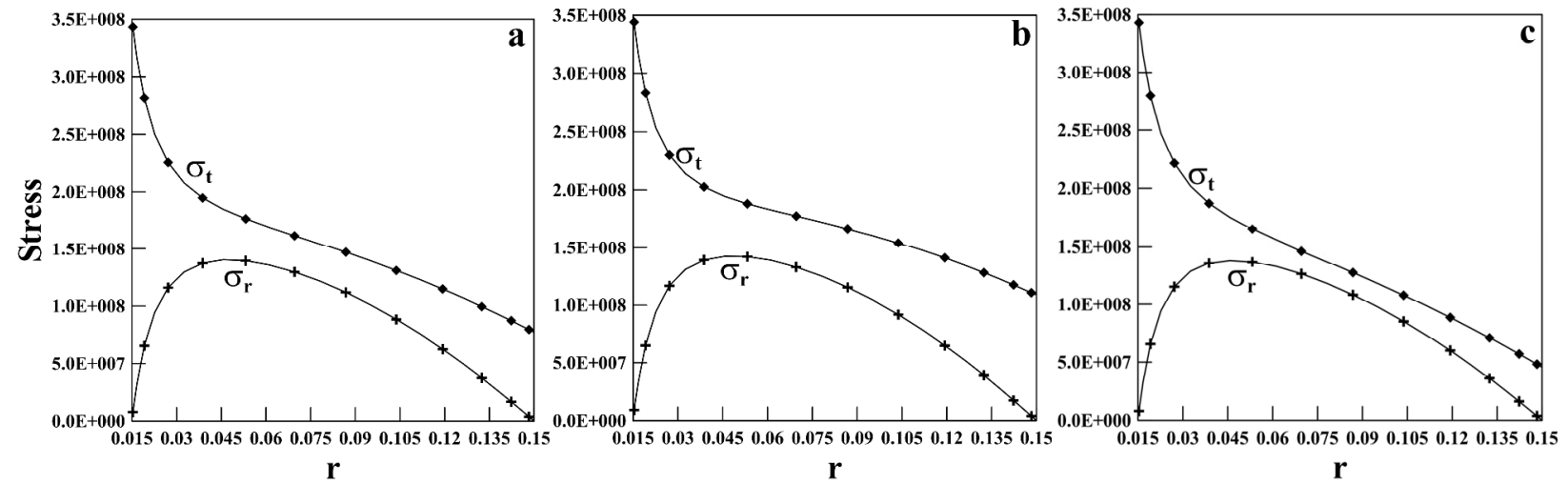

Figure 6. Distribution of radial $\left(\sigma_{r}\right)$ and tangential $\left(\sigma_{t}\right)$ stress field corresponding to yield limit state in an annular disk subjected to (a) pure centrifugal loading due to rotation only, (b) combined loading with higher inner surface temperature $\left(T_{a}\right)$ and (c) combined loading with higher outer surface temperature $\left(T_{b}\right)$.

To explore the phenomenon in greater detail radial $\left(\sigma_{r}\right)$ and tangential $\left(\sigma_{t}\right)$ stress fields of an annular disk $(a / b=0.8)$ is plotted in Figure 6(a) to (c) corresponding to the yield limit state, attained by centrifugal and the two types of thermal loading. Figure 6(a) indicates the stress states corresponding to limit angular speed, and Figures $6(b, c)$ considers temperature field effect corresponding to higher value of inner and outer surface temperature, respectively. The radial stress $\left(\sigma_{r}\right)$ field is almost similar in all the cases but there is a variation in tangential stress $\left(\sigma_{t}\right)$. The reduction in tangential stress field towards the outer radius is most prominent in Figure 6(c), which correspond to the case of higher outer surface temperature $\left(T_{b}\right)$, whereas in Figure 6(b) the decrement is minimum. The tangential stress field coming from pure centrifugal loading, as shown in Figure 6(a), appears to be in an intermediate state. Hence the nature of variation in $\left(\bar{\omega}_{y}\right)$, as observed in Figures 4 and 5 is quite justified for combined centrifugal and thermal loading.

The effect of disk geometries on behavior of thermally loaded disks is also investigated for the normalized limit angular speed variation with temperature and shown again in Figure 7(a) to (d). The individual figures are for particular disk geometry and in each figure the inner surface temperature $T_{a}$ is set to $0^{\circ} \mathrm{C}$ and outer surface temperature $T_{b}$ is varied up to $1500^{\circ} \mathrm{C}$. For all 
temperature distributions the normalized limit angular speed $\left(\bar{\omega}_{y}\right)$ initiate from the same initial value and decreases with increase in the surface temperature of the other boundary. However, for uniform temperature distribution, the curve remains constant. It is also seen that the decrease of normalized limit angular speed is more for exponential temperature distribution, in all the cases of different thickness profiles. Again, when the four figures are compared, it is observed that the initial value of normalized limit angular speed $\left(\bar{\omega}_{y}\right)$ is maximum for exponential disk geometry and minimum for uniform disk geometry.

In Figures 8(a) to (d), the variations in normalized limit angular speed with increasing inner surface temperature $T_{a}$ are also presented for the prescribed disk geometries and temperature distributions. Now, the outer surface temperature $T_{b}$ is set to $0^{\circ} \mathrm{C}$ while the inner surface temperature $T_{a}$ is varied up to $1500^{\circ} \mathrm{C}$. In these figures also, the initial value of normalized limit angular speed is same for all temperature distributions and it increases with increase in the inner surface temperature, except for uniform temperature distribution.
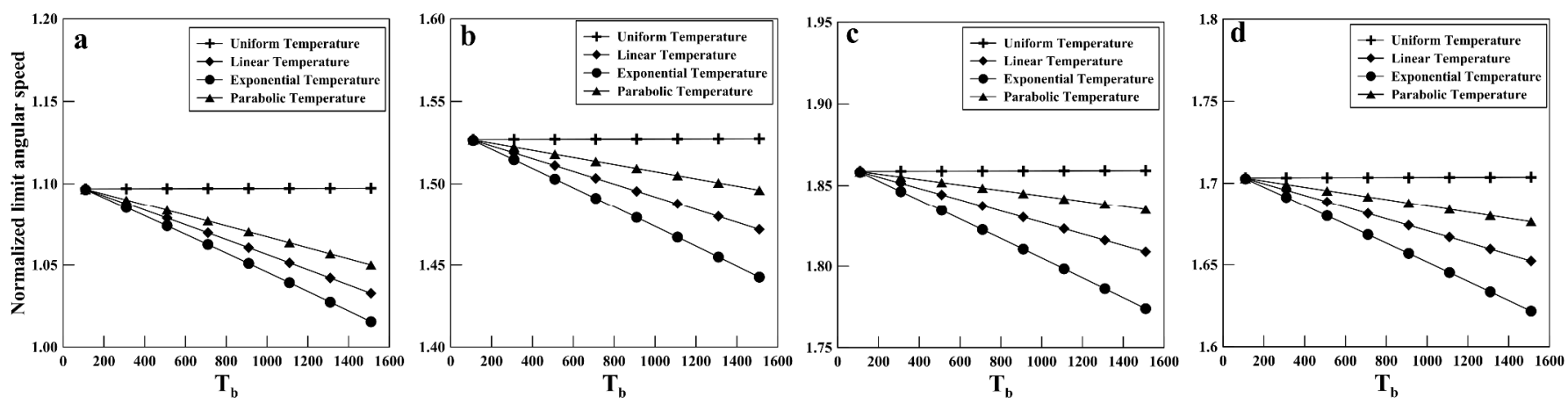

Figure 7. Effect of outer surface temperature distributions $\left(T_{b}\right)$ on normalized limit angular speed $\left(\bar{\omega}_{y}\right)$ for different disk geometries: (a) uniform, (b) taper, (c) exponential and (d) parabolic.
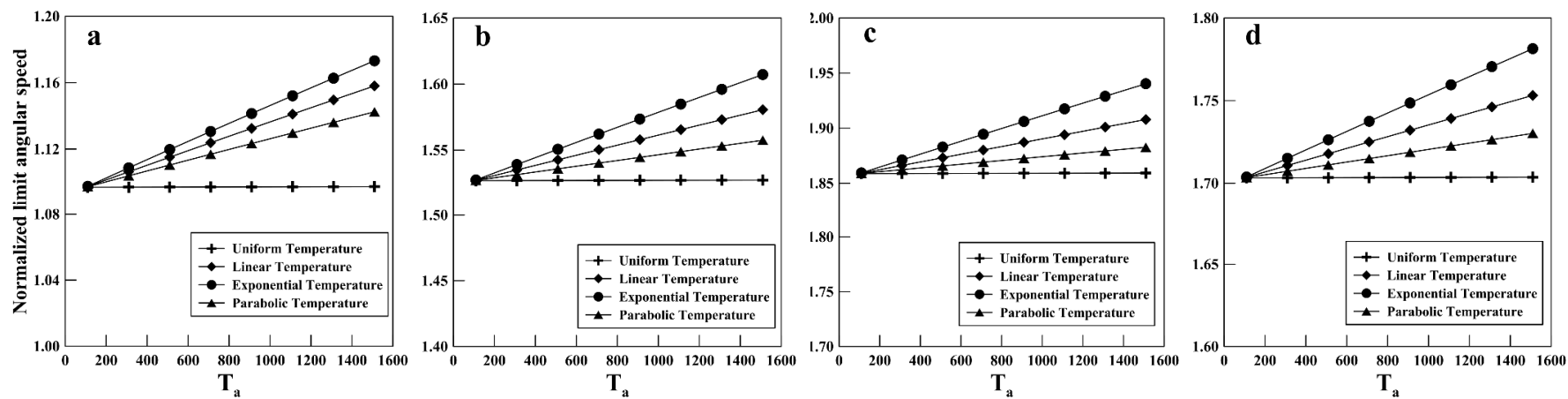

Figure 8. Effect of inner surface temperature distributions $\left(T_{a}\right)$ on normalized limit angular speed $\left(\bar{\omega}_{y}\right)$ for different disk geometries:. (a) uniform, (b) taper, (c) exponential and (d) parabolic.
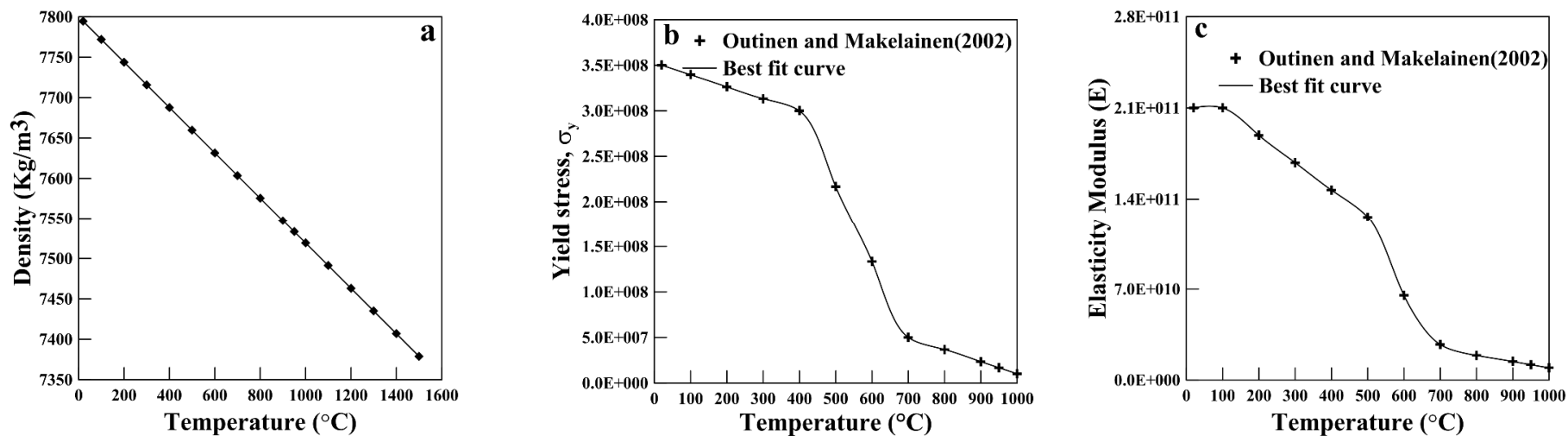

Figure 9. Variation of disk material properties $\rho, \sigma_{y}$ and $E$ with temperature: (a) density $(\rho)$, (b) yield Stress $\left(\sigma_{y}\right)$ and (c) Elasticity modulus $(E)$ for structural steel with grade $S 350 G D+Z$ at different specified temperatures. 
In the next part of the study it is assumed that the disk material properties $\rho, \sigma_{y}$ and $E$ are functions of thermal field $T(r)$, and hence limit angular tip speed, $\omega_{y} b$ in $(\mathrm{m} / \mathrm{s})$ is used as the design parameter. The variations in properties are shown in Figure 9 for structural steel with grade $S 350 G D+Z$, following Outinen and Makelainen (2002). The effect of density variation with temperature is obtained by using the relation $\rho(r)=\rho_{o}[1-\{3 \alpha T(r)\}]$, graphical representation of which is shown in Figure 9(a).

The figure corresponds to $\alpha=0.21 \times 10^{-6}{ }^{\circ} \mathrm{C}^{-1}$, but for other values of thermal expansion coefficient, the effect would be different. Variation in yield stress $\left(\sigma_{y}\right)$ and elasticity modulus $(E)$ with temperature field is obtained by using best fit curves on the tabulated values of Outinen and Makelainen (2002).

3.2 Effect of temperature on the variation of density: Figure 10(a) to (d) show the effect of various disk geometries and temperature distributions on the limit angular tip speed $\left(\omega_{y} b\right)$, taking the temperature effect of density variation in to consideration. The inner surface temperature $T_{a}$ is set to $0^{\circ} \mathrm{C}$ and outer surface temperature $T_{b}$ is varied up to $1600^{\circ} \mathrm{C}$. As can be seen, with increase in $T_{b}$, the limit angular tip speed decreases for all the temperature distributions. But in case of uniform temperature distribution, the curve remains constant. The temperature effect on density is apparently not much pronounced in Figures 10(a) to (d), but for higher values of thermal expansion coefficient, the effect is found to be more.

The effect of density variation with temperature for the prescribed disk geometries and temperature distribution are also depicted in Figure 11(a) to (d). In this case, the outer surface temperature $T_{b}$ is set to $0^{\circ} \mathrm{C}$ and the limit angular tip speed variation with increasing inner surface temperature $T_{a}$ is plotted. The variation of density is governed by the same relation of the previous case, as shown in Figure 9(a). As the inner surface temperature increases the limit angular tip speed increases except for uniform temperature distribution just like in the previous case.
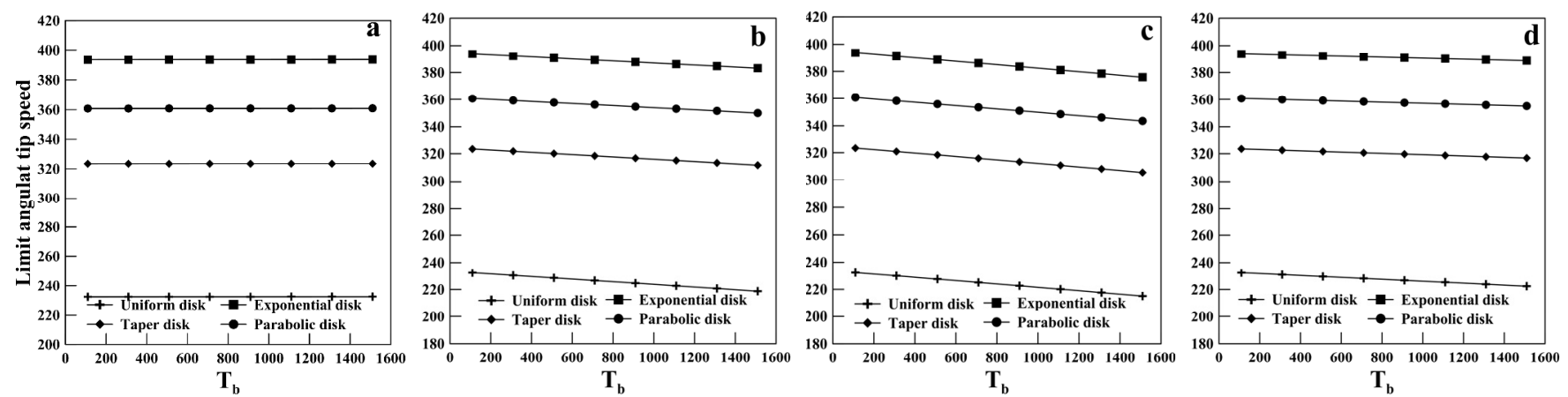

Figure 10. Effect of density variation on limit angular tip speed for different temperature distributions of outer surface $T_{b}$ : (a) uniform, (b) linear, (c) exponential and (d) parabolic temperature.
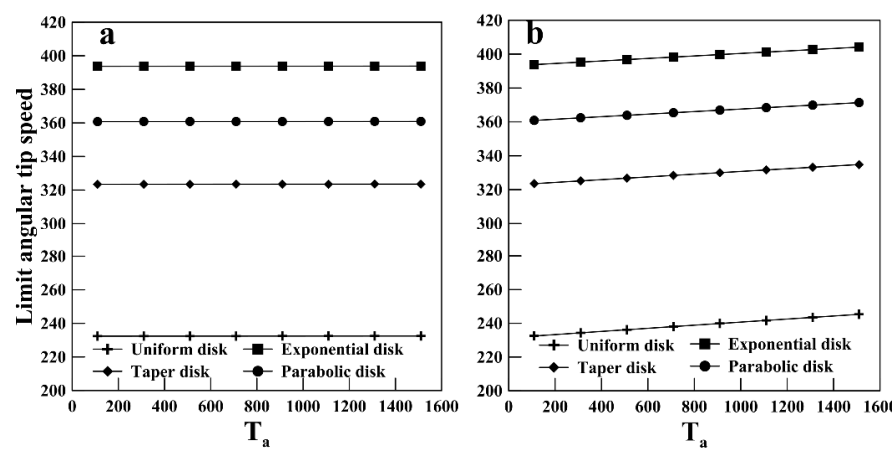

$T_{a}$
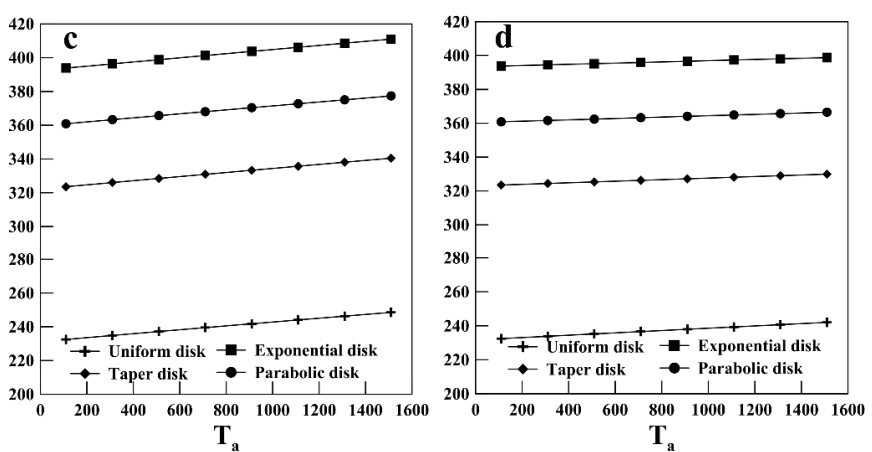

Figure 11. Effect of density variation on limit angular tip speed for different temperature distributions of inner surface $T_{a}$ : (a) uniform, (b) linear, (c) exponential and (d) parabolic temperature.

3.3 Effect of temperature on yield stress: The effect of temperature on yield stress is studied considering other material property values $E, \alpha, \rho$ and $\nu$ as constant. The analysis is carried out for four different temperature distribution profiles and disk profiles. For each of the non-uniform temperature distribution profiles as presented graphically in Figure 2(a), the temperature boundary condition is assumed as $T_{a}=0^{\circ} \mathrm{C}$ and $T_{b}=1000{ }^{\circ} \mathrm{C}$. The variation of yield stress with temperature is already reported in Figure 9(b). Figures 12(a) and (b) shows the variation of yield stress with the disk radius using $T_{b}$ as a parameter for a disk with linear and 
parabolic temperature distributions. The figures are derived from the temperature variation with radius and yield stress variation with temperature, i.e., from the combination of Figure 2(a) and Figure 9(b). It can be seen that in both the cases the yield stress decreases as radius of the disk increases and outer surface temperatures increases.
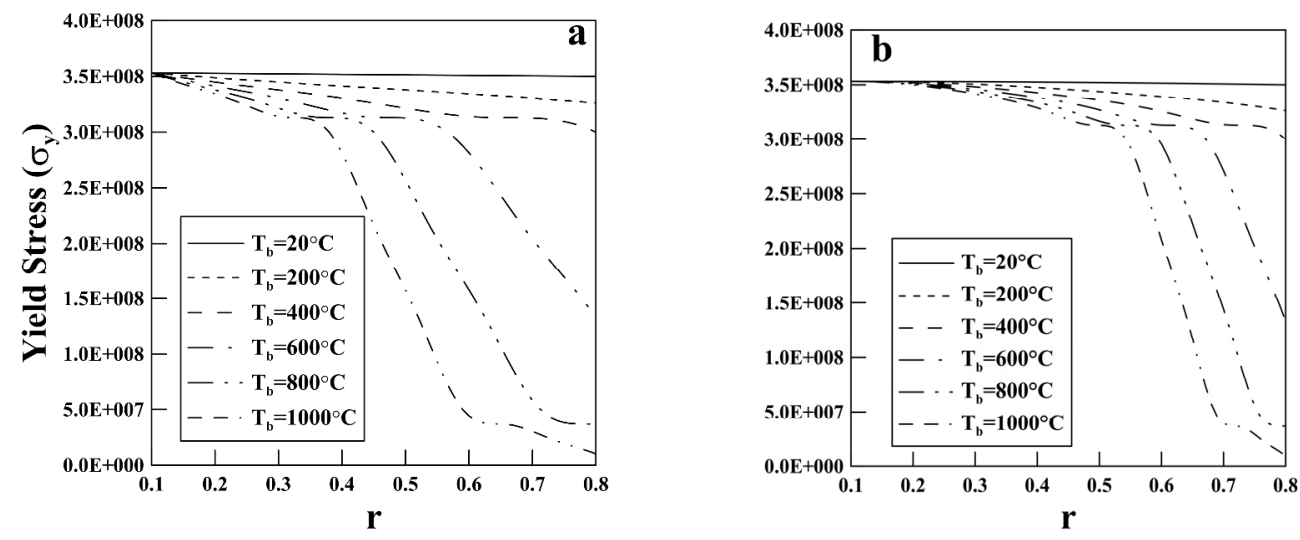

Figure 12. Yield Stress variation in the disk for (a) linear temperature distribution and (b) parabolic temperature distribution.
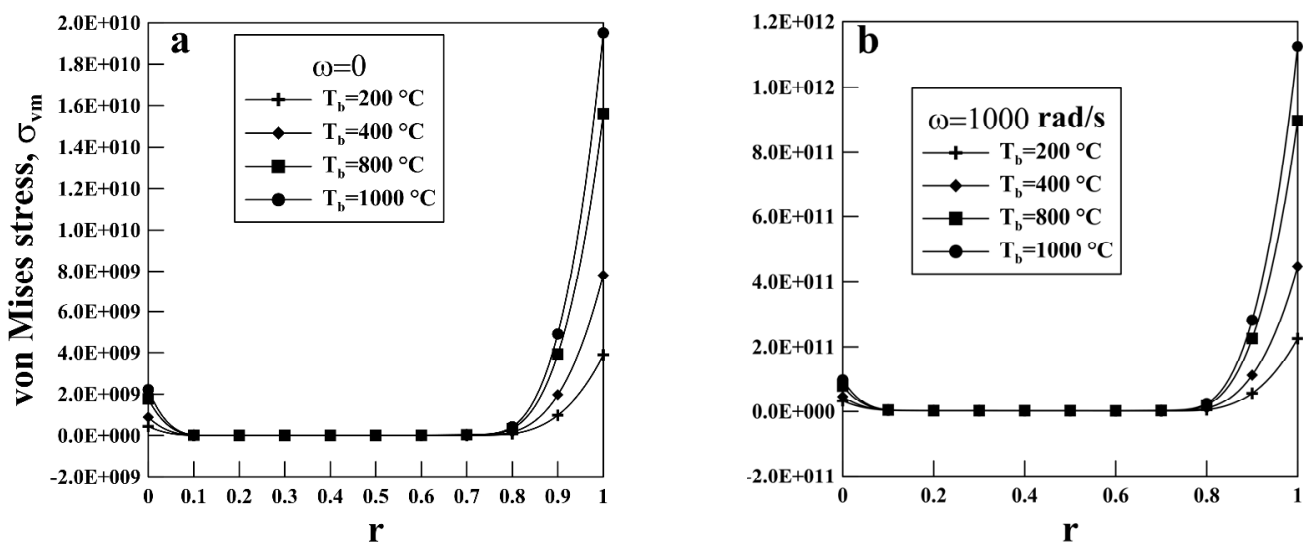

Figure 13. Variation of von Mises stress $\left(\sigma_{v m}\right)$ for parabolic temperature distribution profile for uniform disk at (a) $\omega=0$ $\mathrm{rad} / \mathrm{s}$ and (b) $\omega=1000 \mathrm{rad} / \mathrm{s}$, at four different outer surface temperatures $\left(T_{b}\right)$.
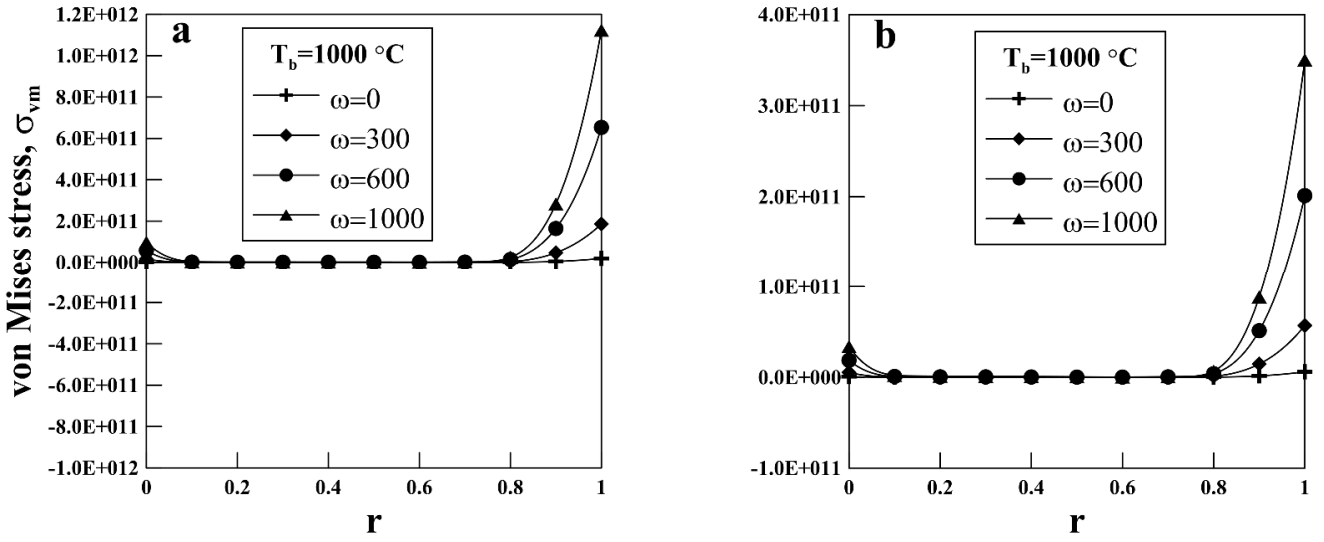

Figure 14. Variation of von Mises stress ( $\left.\sigma_{v m}\right)$ for parabolic temperature distribution profile for (a) uniform disk and (b) parabolic disk, at four different angular speeds. 
The effect of temperature variation on von Mises stress is studied first and the stress distribution with radius is plotted in Figure 13(a) and (b) for uniform disk with parabolic temperature distribution profile. The study is carried out for two different angular speeds $a=0 \mathrm{rad} / \mathrm{s}$ and $a=1000 \mathrm{rad} / \mathrm{s}$ respectively. It is observed that von Mises stress $\left(\sigma_{v m}\right)$ first decreases towards the inner radius of the disk and then remains constant for all the specified outer surface temperatures and again there is an increase in von Mises stress $\left(\sigma_{v m}\right)$ towards the outer radius of the disk. It is also observed that the induced von Mises stress profile increase with outer surface temperature values. For the two different cases of static and rotating disk, the von Mises stress is higher for the rotating one, being apparent in Figure 13(b).

In the next study, the variation of von Mises stress for different values of angular speeds is considered and shown in Figure 14(a) and (b). In this case, temperature distribution is assumed to be parabolic and the two figures present results for uniform and parabolic thickness disks. It is observed that von Mises stress $\left(\sigma_{v m}\right)$ increases with increase in angular speeds but the increase is significantly lower in parabolic disk geometry as compared to uniform disk geometry.
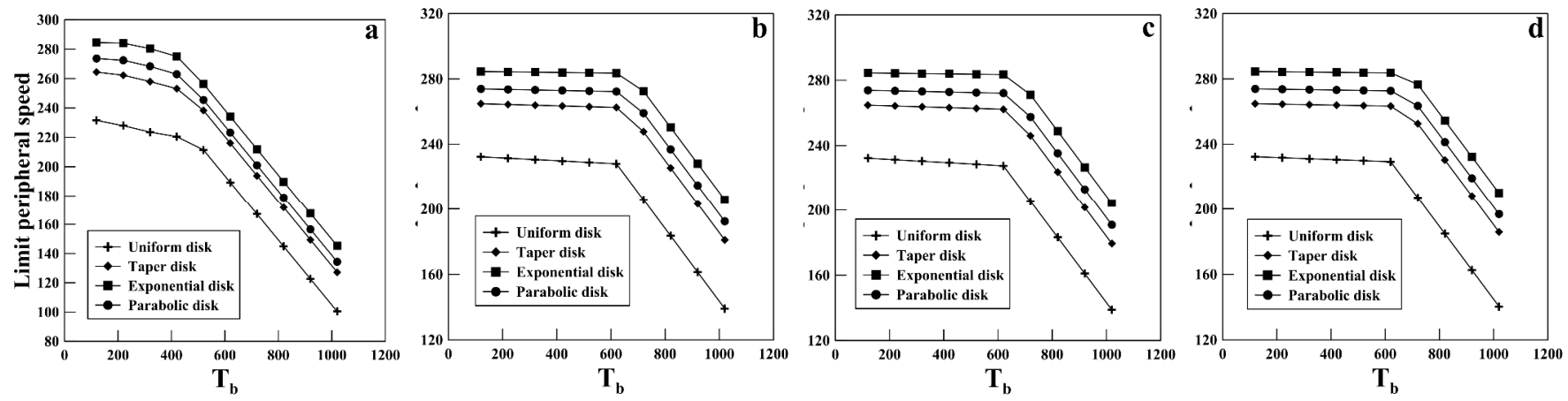

Figure 15. Plots of limit peripheral speed with outer surface temperature $\left(T_{b}\right)$ having different temperature distributions: (a) uniform, (b) linear, (c) exponential and (d) parabolic temperature.

A comparison of the induced stress profile of von Mises stress $\left(\sigma_{v m}\right)$ due to thermo-mechanical loading with the allowable yield stress profile (as shown in Figure 12) would establish the limit angular speed for any given temperature distribution and boundary conditions. This angular speed is not amenable for normalization by the parameter $\omega_{y} b \sqrt{\rho / \sigma_{y}}$, as $\sigma_{y}$ is a field variable here. Hence this speed is called as limit peripheral speed and is presented in dimensional form. Figure 15(a) to (d) show the variation of limit peripheral speed with $T_{b}$ for different disk geometries and temperature distributions. From these figures it is illustrated that, there is a fall of limit peripheral speed with increase in outer surface temperature and this reduction is more prominent after $400{ }^{\circ} \mathrm{C}$. The observation is supported by the fact that beyond this temperature the yield stress also falls rapidly with temperature, as shown in Figure 12.
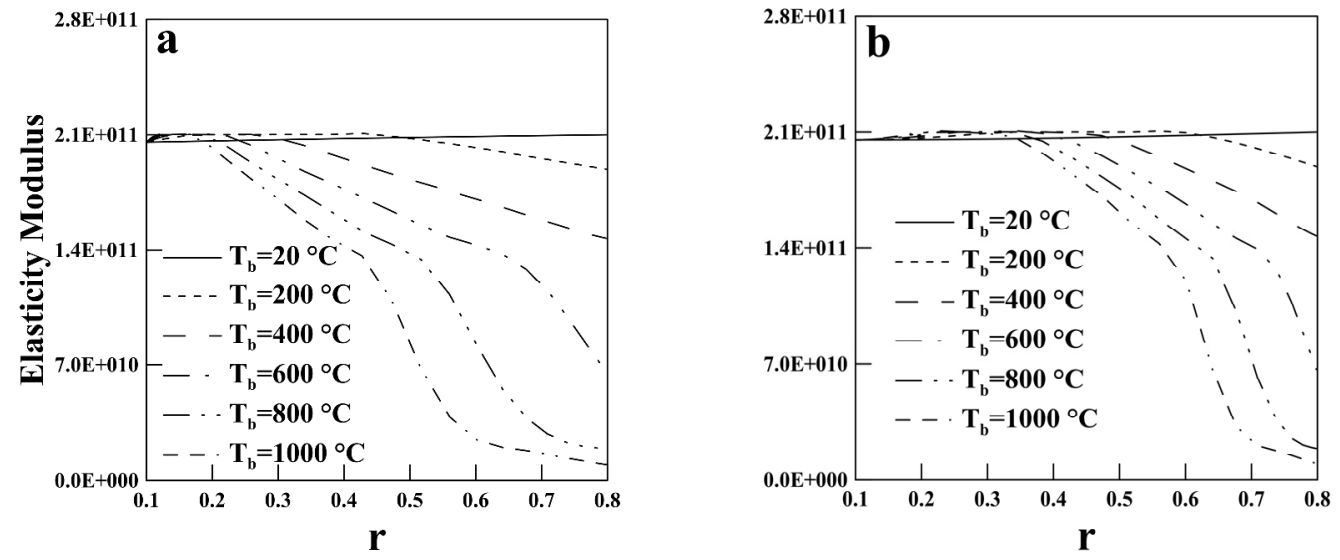

Figure 16. Variation of Elasticity modulus along the radius of the disk having different temperature distributions (a) linear and (b) parabolic temperature. 
3.4 Effect of temperature on elasticity modulus: The effect of temperature on elasticity modulus is studied considering the numerical values of the system parameter as $\sigma_{y}=350 \mathrm{MPa}, v=0.3, \rho=7800 \mathrm{~kg} / \mathrm{m}^{3}$ and $\alpha=0.21 \times 10^{-6}{ }^{\circ} \mathrm{C}^{-1}$. For each of the non-uniform temperature distribution profile as presented graphically in Figure 2(a), the temperature boundary condition is assumed as $T_{a}=0^{\circ} \mathrm{C}, T_{b}=1000{ }^{\circ} \mathrm{C}$. The variation of elasticity modulus with temperature is obtained from the experimental values reported by Outinen and Makelainen (2002) and presented earlier in Figure 9(c).

The variation of elasticity modulus with radius is shown in Figure 16(a) and (b) for a disk having linear and parabolic temperature distributions at various specified outer surface temperatures. It could be observed that for both cases the elasticity modulus declined with radius and this trend becomes more predominant with increase in the values of outer surface temperatures.

Figure 17(a) to (d) indicates the variations of normalized limit angular speed $\left(\omega_{y} b \sqrt{\rho / \sigma_{y}}\right)$, with outer surface temperature $T_{b}$ for different disk geometries and in each figure the effect is shown for four different temperature distributions. These figures indicate fall of normalized limit angular speed with increasing outer surface temperatures except for uniform temperature distribution, where it remains constant. The reduction is more prominent in exponential temperature distribution. It may be noted that $\bar{\omega}_{y}$ has been used as design parameter because density and yield stress is assumed to be constant in this analysis.
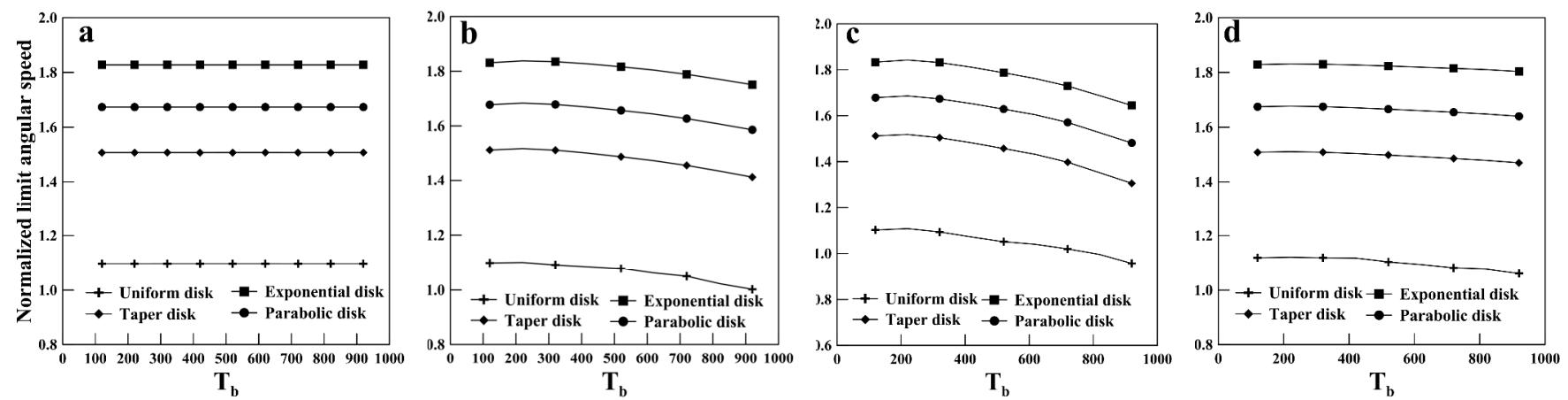

Figure 17. Plots of normalized limit angular speed with outer surface temperature $\left(T_{b}\right)$ having different temperature distributions: (a) uniform, (b) linear, (c) exponential and (d) parabolic temperature.

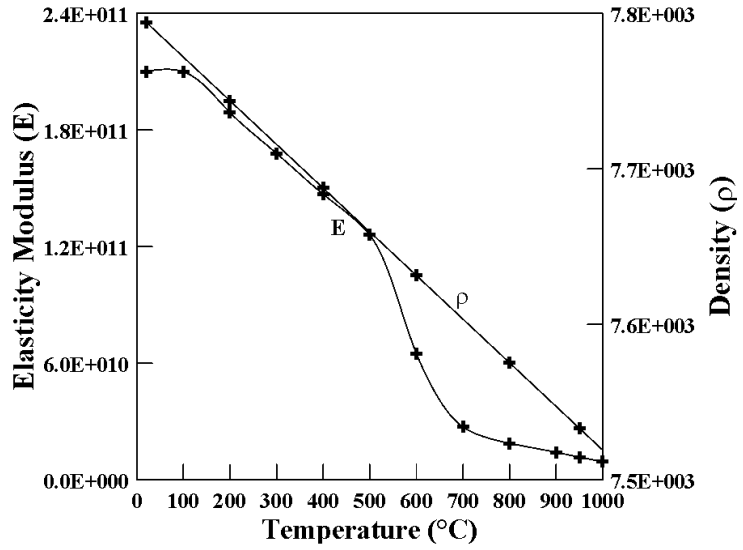

Figure 18. Simultaneous variation of density and elasticity modulus $(E)$ with temperature.

3.5 Effect of temperature on simultaneous variation of density and elasticity modulus: The influence of temperature on both density and elasticity modulus is considered in this section with the same variation of density and elasticity modulus with temperature, as considered previously. The simultaneous variation of density and elasticity modulus with temperature is presented in Figure 18 once again, being the simultaneous plots of Figures 9(a) and 9(c) up to $1000^{\circ} \mathrm{C}$.

The influence of temperature on both density and elasticity modulus with temperature for the prescribed disk geometries and temperature distribution are depicted in Figures 19(a) to (d). In this case, the temperature boundary condition is estimated at $T_{a}=0^{\circ} \mathrm{C}$, and outer surface temperature $T_{b}$ is varied up to $1000^{\circ} \mathrm{C}$. The variation of limit peripheral tip speed $\omega_{y} b$ with increasing outer surface temperature $T_{b}$ is plotted, for each of the four temperature distribution profiles. The effects of four different disk geometries are presented in each of these figures. There is a decrease in limit peripheral speed when outer surface 
temperature increases but exception exists for uniform temperature distribution. The decrease of limit peripheral speed is more for exponential temperature distribution, in line with the observations of previous cases, but the effect is slightly more prominent due to the combined influence of temperature on both density and elasticity modulus.
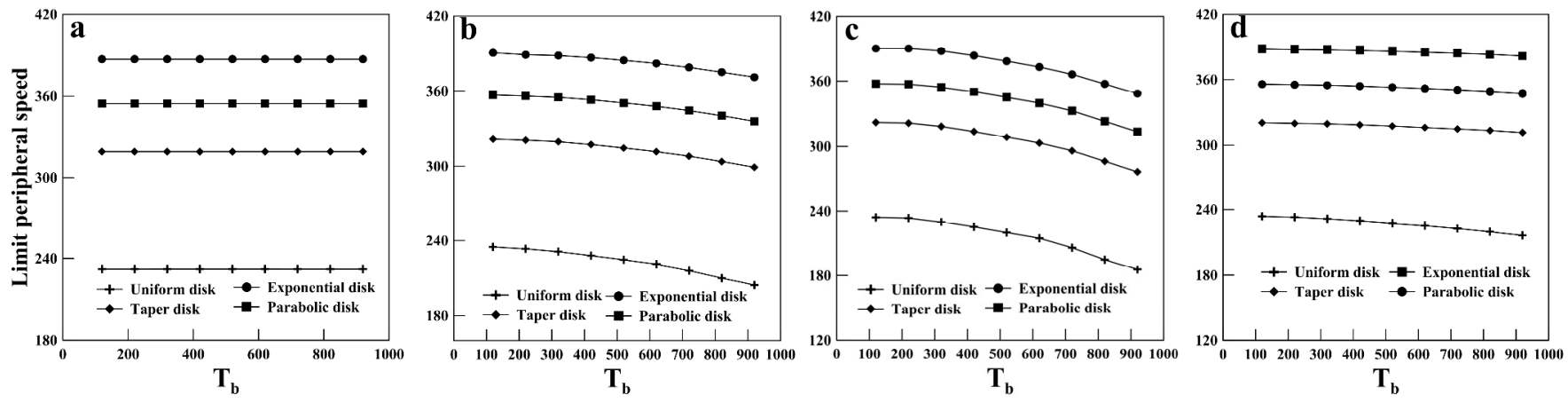

Figure 19. Plots of limit peripheral speed with outer surface temperature $\left(T_{b}\right)$ having different temperature distributions: (a) uniform, (b) linear, (c) exponential and (d) parabolic temperature.

Finally, 3D and contour plots of von Mises stress showing its simultaneous variation with rotational speed and temperature for four different disk geometries is presented in Figures 20 (a) to (d). In these figures, temperature field is assumed to have linear variation, with the temperature boundary conditions: $T_{a}=0^{\circ} \mathrm{C}$ and $T_{b}=$ prescribed. However, the physical parameters of the disk material are assumed to be constant, in these plots. It is observed that with increase in $T_{b}$ as well as rotational speed, von Mises stress increases, but thermal effect is rather insignificant. Another set of plots with high value of thermal co-efficient would reveal the thermal stress effect, but such a plot is omitted to maintain brevity.

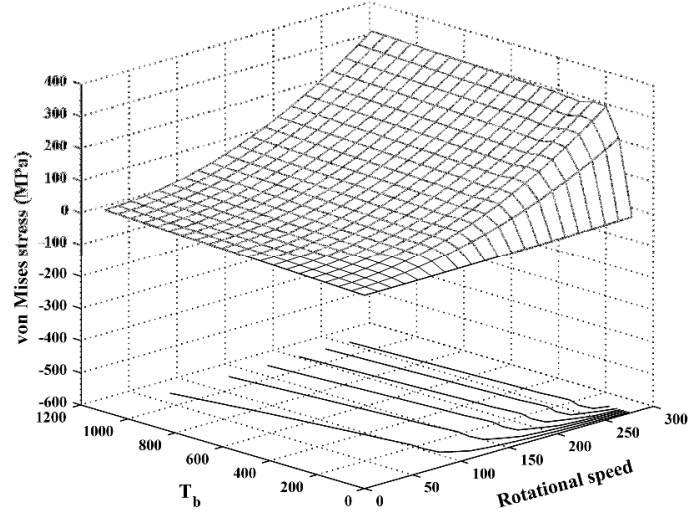

(a)

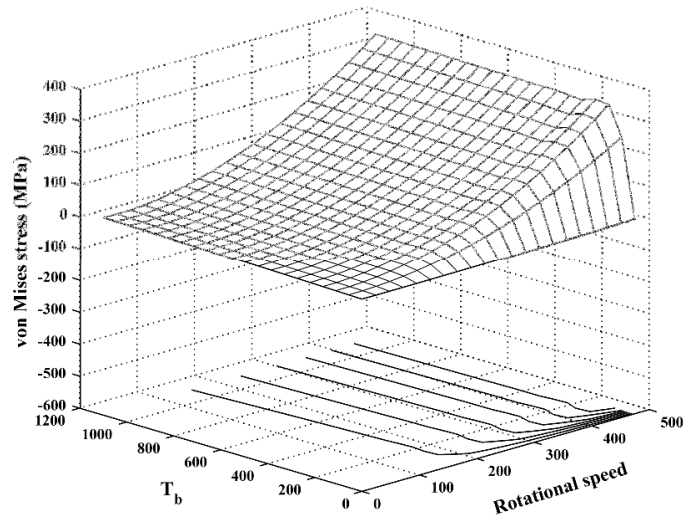

(c)

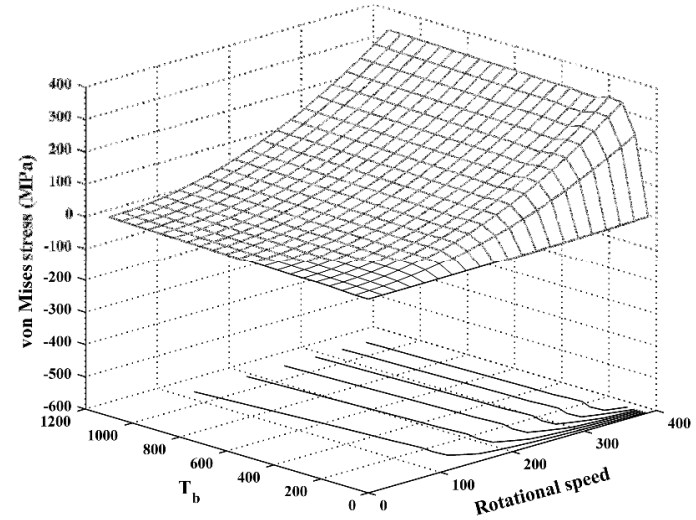

(b)

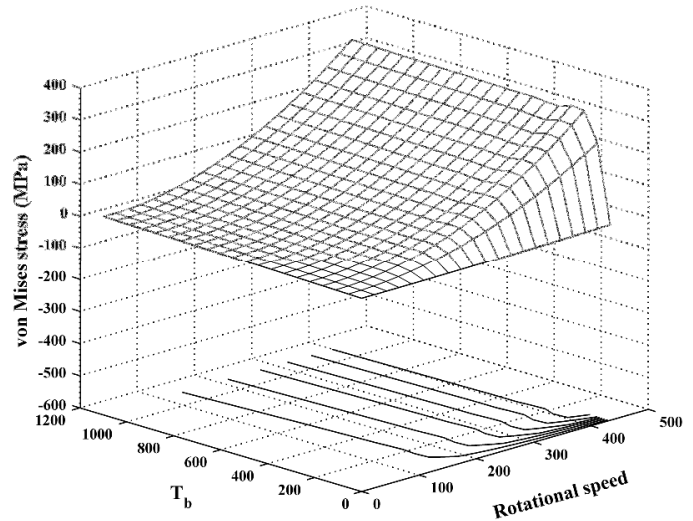

(d)

Figure 20. 3D and contour plots of von Mises stress showing it's variation with rotational speed $(\mathrm{rad} / \mathrm{sec})$ and $T_{b}\left({ }^{o} C\right)$ for various disk geometries: (a) uniform, (b) taper, (c) exponential and (d) parabolic. 


\section{Conclusions}

This paper investigates the rotating disk behavior for combined thermal load and rotational inertia effects by using an approximate solution. The estimated solution of the unknown displacement field from the governing equation is obtained by supposing a series estimation following Galerkin's principle. The proposed method has been validated successfully with published material and relatively good agreement is documented. The influence of disk geometry and temperature field variation on the performance of rotating disks is considered and normalized value of limit angular speed is furnished. Temperature effect on yield stress is also studied and the limit peripheral speed for any given temperature distribution and boundary conditions is established under thermo-mechanical loading in dimensional form. Also the influence of temperature field on the material properties, including density, elasticity modulus as well as combination of both density and elasticity modulus is considered in the present paper and the numerical results are furnished. A future study in this direction would give more understanding of the design of rotating disks using various industrial applications. These results are furnished graphically as design monograms which might prove helpful for the practicing engineers.

\section{Acknowledgement}

The first author is grateful to Council of Scientific \& Industrial Research (CSIR) of the Government of India for the fellowship grant (File No. 09/096(0810)/2014-EMR-I) to carry out the research work.

$\begin{array}{ll}\text { Nomenclature } & \\ a, b & \text { Disk's inner and outer radii } \\ \left\{c_{i}\right\} & \text { Unknown coefficients vector } \\ e_{r}, e_{\theta} & \text { Elastic strain in radial and tangential directions } \\ E & \text { Material's elasticity modulus } \\ \{f\} & \text { Load vector } \\ h_{0}, h & \text { Thickness at the root and thickness at any radius of the disk } \\ {[K]} & \text { Stiffness matrix } \\ n, k & \text { Parameters that control the disk's thickness } \\ r, \theta, z & \text { variation } \\ \bar{r} & \text { Radial, tangential as well as axial directions } \\ T(r) & \text { Parameter (b-a) } \\ T_{a}, T_{b} & \text { Temperature distribution with radius } \\ u & \text { Disk's inner as well as outer surface temperature } \\ U, V & \text { Radial displacement field } \\ \alpha & \text { Strain energy and disk's work potential } \\ \delta & \text { Coefficient of thermal expansion } \\ \varepsilon_{r}, \varepsilon_{\theta} & \text { Variational operator } \\ \varepsilon^{*} & \text { Total strain in radial and tangential directions } \\ \phi_{i} & \text { Thermal strain } \\ \phi_{o} & \text { Group of orthogonal functions employed for the } \\ v & \text { approximation of displacement field } \\ \rho & \text { Start function of orthogonal set of functions } \\ \sigma_{y} & \text { Poisson's ratio } \\ \sigma_{r}, \sigma_{\theta} & \text { Material's density } \\ \sigma_{v m} & \text { Yield stress of the disk material } \\ \omega & \text { Radial and tangential stresses } \\ \omega_{y} & \text { von Mises' stress } \\ & \text { Disk's dimensional angular speed } \\ & \text { Dimensional value of limit angular speed } \\ & \end{array}$




$\begin{array}{ll}\bar{\omega}_{y} & \text { Normalized limit angular speed, } \omega_{y} b \sqrt{\rho / \sigma_{y}} \\ \omega_{y} b & \text { Limit peripheral speed } \\ \xi & \text { Normalized co-ordinate in radial direction, }(r-a) / \bar{r} \\ (\quad) & \text { Differentiation w.r.t. normalized coordinate } \xi\end{array}$

\section{References}

Bayat M., Saleem M., Sahari B.B., Hamouda A.M.S and Mahdi E., 2008. Analysis of functionally graded rotating disks with variable thickness. Mechanics Research Communications. Elsevier, Vol. 35, pp. 283-309.

Bhowmick S., Misra D. and Saha K.N., 2010. A parametric study of externally loaded rotating disks under shrink fit. Proceedings of the Institution of Mechanical Engineers, Part C: Journal of Mechanical Engineering Science. Professional Engineering Publishing, vol. 224, pp. 2525-2538.

Bhowmick S., Misra D. and Saha K.N., 2010. Variational formulation based analysis on growth of yield front in high speed rotating solid disks. International Journal of Engineering, Science and Technology. Multicraft, Vol. 2, pp. 200-219.

Eraslan A.N., 2003. Elastic-plastic deformations of rotating variable thickness annular disks with free, pressurized and radiallyconstrained boundary conditions. International Journal of Mechanical Sciences. Elsevier, Vol. 45, pp. 643-667.

Gamer U., 1983. Tresca's yield condition and the rotating disk. Journal of Applied Mechanics. Trans ASME, Vol. 50, pp. 676678.

Gamer U., 1984. Elastic-plastic deformation of the rotating solid disk. Ingenieur-Archiv. Springer, Vol. 54, pp. 345-354.

Gamer U., 1985. Stress distribution in the rotating elastic-plastic disk. ZAMM-Journal of Applied Mathematics and Mechanics. Wiley, Vol. 65, pp.136-137.

Hojjati M.H. and Jafari S., 2008. Semi-exact solution of elastic non-uniform thickness and density rotating disks by homotopy perturbation and Adomian's decomposition method Part I: elastic solution. International Journal of Pressure Vessels and Piping. Elsevier, Vol. 85, pp. 871-878.

Nie G.J. and Batra R.C., 2010. Stress analysis and material tailoring in isotropic linear thermoelastic incompressible functionally graded rotating disks of variable thickness. Composite Structures. Elsevier, Vol. 92, pp. 720-729.

Outinen J. and Makelainen P., 2002. Mechanical properties of structural steel at elevated temperatures and after cooling down. Second International Workshop (Structures in Fire), Christchurch.

Peng X.L. and Li X.F., 2010. Thermal stress in rotating functionally graded hollow circular disks. Composite Structures. Elsevier, Vol. 92, pp. 1896-1904.

Rattan M., Chamoli N. and Singh S.B., 2010. Creep analysis of an isotropic functionally graded rotating disk. International Journal of Contemporary Mathematical Sciences, Vol. 5, pp. 419-431.

Rees D. W. A., 1999. Elastic-plastic stresses in rotating disks by von-Mises and Tresca. ZAMM- Journal of Applied Mathematics and Mechanics. Wiley, Vol. 79, pp. 281-288.

Thompson A.S., 1946. Stresses in rotating discs at high temperature. Journal of Applied Mechanics. Trans ASME, Vol. 13, pp.4552.

Tutuncu N. and Temel B., 2009. A novel approach to stress analysis of pressurized FGM cylinders, disks and spheres. Composite Structures. Elsevier, Vol. 91, pp. 385-390.

Vivio F. and Vullo V., 2007. Elastic stress analysis of rotating converging conical disks subjected to thermal load and having variable density along the radius. International Journal of Solids and Structures. Elsevier, Vol. 44, pp. 7767-7784.

Vivio F. and Vullo V., 2008. Elastic stress analysis of non-linear variable thickness rotating disks subjected to thermal load and having variable density along the radius. . International Journal of Solids and Structures. Elsevier, Vol. 45, pp. 5337-5355.

Wauer J. and Schweizer B., 2010. Dynamics of rotating thermoelastic disks with stationary heat source. Applied Mathematics and Computation. Elsevier, Vol. 215, pp. 4272-79. 


\section{Biographical notes}

Priyambada Nayak completed her B.Tech in Mechanical Engineering from College of Engineering and Technology, Bhubaneswar, Odisha in 2007 and Master in Mechanical Engineering in Machine Design specialization from Jadavpur University in 2013. She has worked for an IT industry for two years and also engaged in teaching for two years. Presently she is working as a senior research fellow under CSIR Fellowship. Her current area of research is effect of thermal loading on post elastic behaviour of rotating disk.

Kashinath Saha did his graduation in 1982 from the Mechanical Engineering Department of Jadavpur University. He joined academia as lecturer in 1987, after serving industry for five years. Mr. Saha teaches machine elements and system design in under-graduate level and in the post-graduate level he deals with stress, deformation and dynamic analysis of structural elements. Presently he is working with problems involving geometric and material nonlinearities. He has supervised many research students and has published several journal and conference papers in his academic career.

Received July 2015

Accepted March 2016

Final acceptance in revised form June 2016 